\title{
SIR EDMUND WHITTAKER'S WORK ON AUTOMORPHIC FUNCTIONS
}

\author{
by R. A. RANKIN
}

Although only three of Whittaker's papers $(12,13,14)$ are on the theory of automorphic functions, he retained his interest in it throughout his life. His son, Dr J. M. Whittaker, informs me that his last mathematical conversation with his father was on this subject. The standard English work on automorphic functions by L. R. Ford (4), and its precursor in the Edinburgh Mathematical Tracts series, owe much to Whittaker, and it was he who suggested the term "isometric circle" of which Ford makes such elegant use in his development of the theory. Professor G. N. Watson informs me that Whittaker had expressed his willingness to contribute a chapter on automorphic functions to the revised and expanded edition of Modern Analysis which is in preparation.

One of these three papers $(13,11)$ is concerned with the construction of a function which generalises Weierstrass's $\sigma$-function of elliptic function theory to any group of automorphic functions. Apart from this, Whittaker's main interest in automorphic functions centred round the differential equations that occur in the theory of uniformisation. To indicate the relevance of these differential equations, it is necessary to give a brief account of uniformisation theory.

Let

$$
f(z, u)=0,
$$

be the equation of an algebraic curve, where $f$ is a polynomial in the complex variables $z$ and $u$. The curve is said to be uniformised if functions $Z$ and $U$, meromorphic on a certain domain $D$, can be found such that every pair of solutions $z, u$ of (1) is given by

$$
z=Z(t), \quad u=U(t)
$$

for some $t \in D$. Since $u$ is, in general, a many-valued function of $z$, it is convenient to regard $z$ as a point on a Riemann surface. The nature of the uniformising functions depends upon the genus $p$ of this surface.

If $p=0$, the equation (1) can be uniformised by rational functions $Z$ and $U$, while, if $p=1$, elliptic functions are required. In these cases the uniformisation problem can be regarded as completely solved, in the sense that the functions $Z$ and $U$ can be explicitly constructed.

If $p \geqslant 2$, the nature of the problem changes. Uniformising functions $Z$ and $U$ exist, but they are automorphic functions belonging to a group of bilinear transformations

$$
t_{S}=\frac{a t+\beta}{\gamma t+\delta}=S(t) \quad(\alpha \delta-\beta \gamma=1),
$$

and satisfy the functional equations

$$
Z\left(t_{S}\right)=Z(t), \quad U\left(t_{S}\right)=U(t)
$$

for all $S^{\prime} \in \Gamma$. The group $\Gamma$ and the associated automorphic functions $Z$ and 
$U$ can be chosen in various ways. In the simplest cases $\Gamma$ possesses the property that its transformations leave invariant a certain fixed circle and its interior, which we may take to be $|t| \leqslant 1$; further, $\Gamma$ is properly discontinuous, which implies that the interior of this circle can be divided up into disjoint fundamental regions, no two of whose points are congruent with respect to transformations of the group $\Gamma$. Such a group is called a Fuchsian group.

The proof that every algebraic equation (1) of genus $p \geqslant 2$ can be uniformised in this way is not easy and involves such deep theorems as Riemann's theorem on conformal mapping. There can be no doubt of the importance of the result, but it should nevertheless be realised that it does not solve the uniformisation problem completely, as most mathematicians tend to assume. The proof ensures the existence of the automorphic functions $Z$ and $U$, but does not construct them; it does not even determine the group $\Gamma$.

It seems to be the case that only one algebraic equation of genus $p \geqslant 2$ has been uniformised explicitly, namely the equation

$$
u^{2}=z\left(z^{4}-1\right) \text {, }
$$

for which $p=2$. The uniformisation was carried through by Burnside (1) in 1893 using results given by Klein and Fricke (6, vol. I, p. 563). The uniformising functions are rational functions of Weierstrass's $\wp$-function regarded as a function of its periods.

For a few other equations, such as (6, vol. I, p. 655 et seq.)

$$
\begin{array}{ll}
u^{2}=z\left(z^{10}+11 z^{5}-1\right) & (p=5), \\
u^{4}+z^{4}=1 & (p=3), \\
u^{8}+z^{8}=1 & (p=21),
\end{array}
$$

and certain transformation equations of elliptic modular function theory, the problem is almost completely solved, in the sense that the groups $\Gamma$ and the automorphic functions are almost completely determined. Thus, for the equations (3), (4) and (5), the groups $\Gamma$ are known normal congruence subgroups of the full modular group $\Gamma(1)$, of indices 120,96 and 384, respectively. In the latter two cases, $z$ can be taken to be $k^{\frac{1}{2}}$ and $k^{\frac{1}{2}}$ respectively, where $k^{2}$ is Legendre's modular function; it then remains to determine the correct branch of $z$ by studying its behaviour under transformations belonging to a representative system of cosets of $\Gamma$ in $\Gamma(1)$.

Nothing, however, is known about the uniformising functions or group of any algebraic equation of genus $p \geqslant 2$ which contains irremovable arbitrary constants. If the group $\Gamma$ of an equation is known, the automorphic functions of that group can be constructed by various methods, for example as quotients of automorphic forms defined by Poincaré series, and the uniformising functions $Z$ and $U$ can then be selected. The problem, therefore, reduces essentially to that of determining $\Gamma$ for a given equation (1).

In Whittaker's long paper (12), which grew out of his Fellowship dissertation and Smith's Prize essay, he developed a method which is particularly applicable 
to hyperelliptic equations, i.e. equations of the form

$$
u^{2}=\left(z-e_{1}\right)\left(z-e_{2}\right) \ldots\left(z-e_{2 p+2}\right)=f(z),
$$

where the constants $e_{n}(1 \leqslant n \leqslant 2 p+2)$ are distinct complex numbers. This equation is of genus $p$ and is of a more general form than might be supposed : for example, every algebraic equation of genus 2 can be expressed in this form. Whittaker showed that the group $\Gamma$ of the equation (6) is a subgroup, of index 2 , in a larger simpler group $\Gamma^{*}$ which is generated by self-inverse transformations, i.e., by elliptic transformations of period 2. The particular advantage of this is that the fundamental region of $\Gamma^{*}$ has genus zero, so that every automorphic function belonging to $\Gamma^{*}$ can be expressed as a rational function of one such automorphic function $z(t)$. In particular, the Schwarzian derivative

$$
\{t, z\}=-\frac{d^{3} z / d t^{3}}{(d z / d t)^{3}}+\frac{3}{2}\left\{\frac{d^{2} z / d t^{2}}{\left(\frac{d z / d t)^{2}}{2}\right.}\right\}^{2}
$$

which is an automorphic function of $\Gamma^{*}$, must be a rational function $R(z)$, and is of the form

$$
R(z)=\frac{3}{8}\left\{\sum_{n=1}^{2 p+2} \frac{1}{\left(z-e_{n}\right)^{2}}-\frac{g(z)}{f(z)}\right\},
$$

where $f(z)$ is defined by $(6)$ and $g(z)$ is a polynomial of degree $2 p$ of the form

$$
g(z)=2(p+1) z^{2 p}-2 p \sum_{n=1}^{2 p+2} e_{n^{2}} z^{p-1}+\sum_{r=0}^{2 p-2} c_{r} z^{2 p-2-r} .
$$

The $2 p-1$ coefficients $c_{0}, c_{1}, \ldots, c_{2 p-2}$ are the so-called accessory parameters and, by a uniqueness theorem of Poincaré, are determined uniquely by the fact that $\Gamma^{*}$ is Fuchsian.

It can be shown that $\Gamma^{*}$ is the monodromic group associated with the differential equation

$$
\frac{d^{2} \eta}{d z^{2}}+\frac{1}{2} R(z) \eta=0 \text {. }
$$

A pair of linearly independent solutions $\eta_{1}, \eta_{2}$ of this equation will change to $A \eta_{1}+B \eta_{2}$ and $C \eta_{1}+D \eta_{2}$, say, on traversing a closed contour round one of its $2 p+2$ singular points $e_{n}$. The group $\Gamma^{*}$ is conjugate to the group generated by the $2 p+2$ self-inverse transformations

$$
t^{\prime}=\frac{A t+B}{C t+D}
$$

Hence, if (7) can be solved, $\Gamma^{*}$ can be found and therefore so can $\Gamma$; the automorphic functions for $\Gamma$ can then be set up and so the equation (6) can be uniformised explicitly. The most important step in this programme is clearly the determination of the accessory parameters.

In 1929 Whittaker returned to this subject (14) and put forward the conjecture that

$$
R(z)=\frac{3}{8}\left[\left\{\frac{f^{\prime}(z)}{f(z)}\right\}^{2}-\frac{2 p+2}{2 p+1} \frac{f^{\prime \prime}(z)}{f(z)}\right]
$$


in the particular case of (6) when *

$$
f(z)=z^{5}+1 \quad(p=2) .
$$

He was led to this conjecture by a consideration of the invariant properties of $R(z)$; for (6) can be transformed into various equivalent forms each of which must yield the same differential equation (7). With this assumption, and for the particular case (9), he solved the associated differential equation (7) in terms of hypergeometric functions and so found $\Gamma^{*}$ and $\Gamma$. He found that $\Gamma^{*}$ is a Fuchsian group and is generated by the five transformations

where

$$
S_{k}(t)=\frac{a t-\epsilon_{k}}{\bar{\epsilon}_{k} t-a} \quad(k=0,1,2,3,4),
$$

$$
a=\left\{\frac{\sqrt{5}+1}{2}\right\}^{\ddagger}, \text { and } \epsilon_{k}=e^{(4 k+1) \pi i / 10} \quad(k=0,1,2,3,4) .
$$

Thus, by Poincare's uniqueness theorem, his conjecture was verified in this case. The group $\Gamma$ is generated by the four transformations $S_{0} S_{1}, S_{0} S_{2}, S_{0} S_{3}$ and $S_{0} S_{4}$; its fundamental region is a curvilinear octagon and the functions associated with it Whittaker termed hyperlemniscate functions. This name was suggested by their being the first known Fuchsian automorphic functions of genus 2, just as the lemniscate functions, which uniformise the algebraic equation $u^{2}=1-z^{4}$ of genus 1 , were the first known elliptic functions. Whittaker did not go so far as to construct his hyperlemniscate functions; in a letter to the writer he remarked that a field as rich as that of elliptic function theory lay open to any mathematician who would do so.

In 1930 Whittaker's pupil Mursi (7) extended this work to the equation

$$
u^{2}=z^{7}+1 \quad(p=3)
$$

and verified the conjecture (8). In the same year Whittaker's son, J. M. Whittaker (14), used the invariant properties of $R(z)$ to verify the truth of (8) in the general hyperelliptic case under the assumption that the accessory parameters are polynomials in the $2 p+2$ numbers $e_{n}$. Again in the same year, D. P. Dalzell (2) showed that, for

$$
u^{2}=f(z)=z^{n}-1 \quad(n \text { even }),
$$

the differential equation (7), with $R(z)$ given by (8), determines a Fuchsian group $\Gamma^{*}$. He also considered a more general differential equation corresponding to the algebraic equation

$$
u^{p}=1-z^{n} \quad(p \text { divides } n) .
$$

He deduced that $\Gamma^{*}$ is Fuchsian from the fact that the differential equation is the same as that differential equation which arises from the $n$th root of one of the Riemann-Schwarz triangle functions. In fact $\Gamma^{*}$ must be a subgroup of the Fuchsian group associated with this triangle function. Being at $\infty$

* This can be included under (6) by taking $p=2$ and one of the branch points $e_{n}$ 
written in a very condensed form, Dalzell's paper has not received the notice due to it. I have recently observed that the use of triangle functions for the uniformisation of algebraic equations, such as (11) and (3), was already noted by Schwarz (10) in 1890 among the Anmerkungen und Zusätze (pp. 363-8) which he appended, in his collected works, to his famous paper of 1873 on hypergeometric functions (p. 211).

Whittaker's method, as developed in (14), was later extended to the general equation

by Dhar (3).

$$
u^{2}=z^{2 n+1}+1
$$

In $1936 \mathrm{~J}$. Hodgkinson (5) considered Whittaker's conjecture for the general equation (6). By using the invariant properties of $R(z)$ he was able to express the accessory parameters in terms of certain incompletely determined symmetric functions of the numbers $e_{n}$, but was unable to prove the conjecture. Since the conjecture applies only to Fuchsian groups, and not to other Kleinian groups in terms of which uniformisation may be possible, it is natural to expect that a proof of its truth for the general case, if such a proof can be constructed, will distinguish at some stage between Fuchsian groups and other groups of transformations. So far no way of doing this has been discovered. It may be noted that Mursi (8) has constructed a differential equation (with $p=1$ ) which does not satisfy the conjecture (8) but yields a Kleinian group having a multiply-connected fundamental region; this group uniformises the equation

$$
u^{2}=\left(z-e_{1}\right)\left(z-e_{2}\right)\left(z-e_{3}\right)\left(z-e_{4}\right) \text {. }
$$

However, Mursi's function $R(z)$ is not symmetric in $e_{1}, e_{2}, e_{3}$ and $e_{4}$, as is necessary in the Fuchsian case.

Thus the truth of the conjecture (8) remains undecided in the general case, although it has been verified for algebraic equations of the forms (10) and (12). It has also been verified in a number of other particular cases associated with the rotation groups of the regular solids (9).

It is likely that Whittaker's work on differential equations and special functions grew out of his interest in the differential equations that have been described. As has been stated, he retained his interest in the theories of automorphic functions and uniformisation throughout his life, but, as his interest in these subjects was a practical one, he probably felt rather out of sympathy with the increasingly abstract approach which has become fashionable since the publication in 1913 of H. Weyl's Die Idee der Riemannsche F'äche. His viewpoint is indicated by a sentence in his paper (14) of 1929 in which he remarks that " the progress of the theory has been arrested at this point for a whole generation " in reference to the fact that no general method of determining the accessory parameters has yet been found. Although the theory has developed in other directions during the intervening years, there is no doubt that a solution of Whittaker's problem would give it new impetus. 


\section{REFERENCES}

(1) W. Burnside, Note on the equation $y^{2}=x\left(x^{4}-1\right)$, Proc. London Math. Soc. (1), 24 (1893), 17.20.

(2) D. P. Dalzell, A note on automorphic functions, J. London Math. Soc., 5 (1930), 280-2.

(3) S. C. Dhar, On the uniformisation of a special kind of algebraic curve of any genus, $J$. London Math. Soc., 10 (1935), 259-263.

(4) L. R. Ford, Automorphic functions (New York, 1929).

(5) J. Hodgkinson, Note on the uniformization of hyperelliptic curves, $J$. London Math. Soc., 11 (1936), 185-192.

(6) F. Klein and R. Fricke, Vorlesungen über die Theorie der elliptischen Modulfunktionen (Leipzig, 1890-2).

(7) M. Mursi, On the uniformisation of algebraic curves of genus 3, Proc. Edinburgh Math. Soc. (2), 2 (1930), 101-7. $166-9$

(8) M. Mursi, A note on automorphic functions, J. London Math. Soc., 6 (1931),

(9) R. A. Rankin, The differential equations associated with the uniformization of certain algebraic curves, Proc. Roy. Soc. Edin., A 65 (1958), 35-62.

(10) H. A. Schwarz, Gesammelte mathematische Abhandlungen, vol. 2 (Berlin, $1890)$.

(11) H. Stahl, Berechtigung einer Arbeit von Herrn E. T. Whittaker, Arch. Math. Phys. (3), 10 (1906), 336-7.

(12) E. T. Whittaker, On the connexion of algebraic functions with automorphic functions, Phil. Trans., 192A (1898), 1-32.

(13) E. 'T. Whittaker, Note on a function analogous to Weierstrass' sigmafunction, Mess. Math., 31 (1902), 145-8.

(14) E. T. Whittaker, On hyperlemniscate functions, a family of automorphic functions, $J$. London Math. Soc., 4 (1929), 274-8.

(15) J. M. Whittaker, The uniformisation of algebraic curves, J. London Math. Soc., 5 (1930), 150-4.

Department of Mathematics

The UnIVERsity

GLasgow 